\title{
Estimation of a joint distribution for several phenotypic traits in breeding or ancient populations
}

\author{
Anna A. Igolkina \\ SPbPU, St.Petersburg, Russia \\ igolkinaanna11@gmail.com
}

\author{
Sergey Nuzhdin \\ SPbPU, St.Petersburg, Russia \\ UCS, Los Angeles, USA \\ snuzhdin@usc.edu
}

\author{
Maria G. Samsonova \\ SPbPU, St.Petersburg, Russia \\ m.g.samsonova@gmail.com
}

\begin{abstract}
Prediction of phenotypic trait values for a population is a challenge in genomic selection and evolutionary studies. We propose a pipeline of developed models, which estimates the distribution of several trait values for a population with known allele (SNP) frequencies. The pipeline consists of two parts: mtmISEM model, which describes the influences of pleiotropic and single-trait SNPs on genetically correlated traits, and dipophen method, which predicts the distribution of traits in a population, based on mtmlSEM. As an input, the pipeline gets allele frequencies evaluated for a breeding population or SNP ancestral population. The pipeline was applied to two datasets: one from the breeding soybean project and the other from the chickpea "trade routes" project. While allele frequency in the breeding population can be directly calculated, for estimating allele frequencies in the ancestral population, we developed new models.
\end{abstract}

Keywords - distribution of traits, allele frequencies, pleiotropic SNPs, SEM, Bernoulli random variables, population admixture

\section{INTRODUCTION}

\section{Motivation}

Significant determinants for many phenotypic traits are stored in the genome. The standard way to identify genetic variants (SNPs) associated with phenotypic traits considers them independently; however, traits are often genetically correlated and should be considered in GWAS analysis simultaneously. Recent methods to cope with correlated traits do not separate SNPs into pleiotropic and single-trait effects; therefore, we propose the mtmlSEM model, which structures hidden biologics mechanism affecting correlated traits and directly searches SNPs of different effects.

The mtmlSEM model not only allows us identifying SNPs associated with related phenotypic variables but also provides the technique to predict phenotypic traits for a new genotype.

The prediction of phenotypic traits for an individual is not as impressive as for the whole population. For the latter, we propose the fast method, dipophen, to predict the joint distribution of several phenotypic traits. This method takes as input allele frequencies in a population and parameter estimated from any linear genotype-phenotype model (e.g., mtmlSEM).

In breeding studies, the evaluation of allele frequencies for a given population is quite simple and requires SNP data for representative samples. In evolutionary studies, estimation of SNP frequencies in ancestral populations requires mathematical modeling. The problem in such modeling is that frequencies belong to a compositional data class (sum of frequencies equal to 1). Still, none of the models, which predict allele frequencies in populations, consider this compositional nature, and new, more correct models are required.

Aim

This study aims to develop the pipeline to estimate the joint distribution of genetically correlated traits in populations characterized by SNP frequency data. To reach the goal, the following list of tasks to develop was set:

- genotype-phenotype model based on structural equation modeling, which describes the biosocial mechanism affecting genetically correlated traits, and separate SNPs of pleiotropic and single-trait effect.

- $\quad$ statistical technique to estimate the joint distribution of the weighted sum of independent and non-identically Bernoulli random variables.

- Bayesian model to estimate allele frequency in the ancestral population, which considers possible ways of dispersal of the population from the center towards cultivation locations.

\section{METHODS}

mtmlSEM model

To describe genetically correlated phenotypes we proposed the following mtmlSEM (multi-trait, multi-locus) model (Fig 1A):

$$
\begin{aligned}
& \eta=B \eta+\Pi g+\varepsilon \\
& p=\Lambda \eta+\mathrm{K} y+\delta
\end{aligned}
$$

Where $p$ is a vector of phenotypes, $\eta$ is a vector of latent factors, $g$ and $y$ are SNP variables of pleiotropic and singletrait effects, respectively; $\Pi$ and $\mathrm{K}$ are matrixes of SNP influences on latent factors and phenotypes, respectively $\Lambda$ is a matrix of factor loadings, Bis a matrix of relationships between latent factors, $\varepsilon$ and $\delta$ are random errors. We developed an automatic algorithm to specify the model, and Bayesian scheme to get parameter estimates. We utilized the symopy package [1] and mtmISEM model [2].

\section{Estimation of the joint distribution of several traits}

A mtmlSEM model can be used to predict phenotype from genotype in the following manner:

$$
\hat{p}=\Lambda(1-B)^{-1} \Pi g+K y,
$$

so that each phenotype is a linear combination of SNPs, each taking values from $\{0,1,2\}$ (additive model). To estimate the distribution of phenotypes in a population, we 
can use allele frequencies of $g$ and $y$ and set that random variable $\tilde{p}$ is:

$$
\tilde{p}=2\left(\Lambda(1-B)^{-1} \Pi \tilde{g}+K \tilde{y}\right),
$$

where $\tilde{g}$ and $\tilde{y}$ are vectors of independent Bernoulli random variable with population allele frequencies. To estimate the joint distribution of $\tilde{p}$, we developed the dipophen method, which utilizes the bernmix package (https://github.com/iganna/bernmix). The bernmix package allows estimating the distribution of a linear combination of Bernoulli random variables based on Fourier transform and faster than analogs.
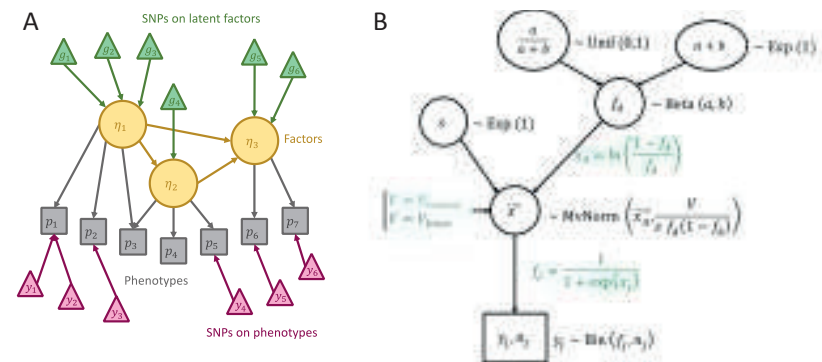

Fig. 1. (A) mtmlSEM model; (B) Bayesian model to predict allele frequencies within clusters

\section{Prediction of allele frequencies of ancestral chickpea populations}

We utilized the chickpea genotyped landraces from Vavilov's collection: landraces form ten clusters, each composed of geographic locations with genotypes samples. We examined two hypothetical dispersals of the chickpea population within each cluster and estimated allele frequencies in their centers. We hypothesized that chickpea was spread within a cluster along the binary path either in line with trade routes or by diffusion-like manner. In the model, the contrast between these hypotheses was reflected by different given covariance matrices V (Fig 1B).

The model's important feature is the use of compositional data analysis in the application to allele frequency changes along the binary path from the center towards locations: we considered changes in balances between allele frequencies instead of direct frequencies (Fig 1B).

\section{RESULTS}

\section{Soybean: breeding pairs}

We analyzed 257 soybean accessions (Glycine max) with known genotypes (4767 SNPs) and values of three traits: productivity, oil and protein contents. We transform ilrtransformation [3] to oil and protein contents, specify the mtmlSEM model with one latent variable, and get parameter estimates. Then we tested all possible pairs of soybean accessions as potential breeding pairs. For each pair, we computed SNP frequencies in F2 and predicted the joint distribution of three phenotypes (Fig 2). The results allowed us to rank all pairs according to the probability of having efficient offspring in F2.
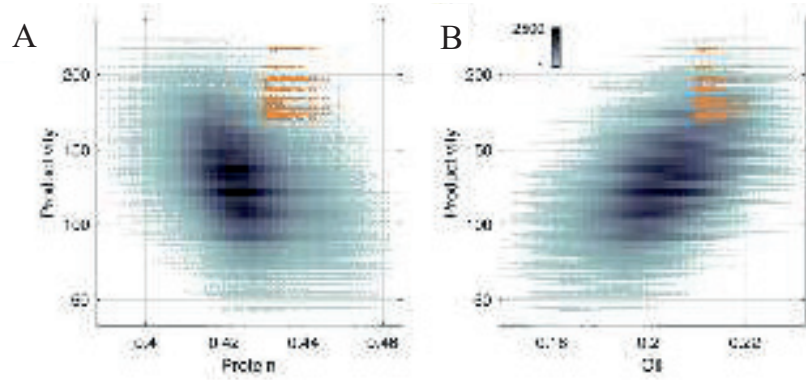

Fig 2. Distributions of trait values in all possible homozygotes. Shades of blue display the density of homozygotes on scatterplots. Orange color denote homozygotes, which satisfy introduced thresholds: productivity should be higher than 170 , protein content - higher than 0.43 , oil content - higher than 0.21

\section{Chickpea: ancestral populations}

The chickpea dataset consisted of 421 samples genotyped on 2579 SNPs and phenotyped on 16 traits. Samples were attributed to ten geographically justified populations. First, we specified mtmlSEM model and obtained 5 latent factors, which describe correlation in 16 traits (Fig. 3).

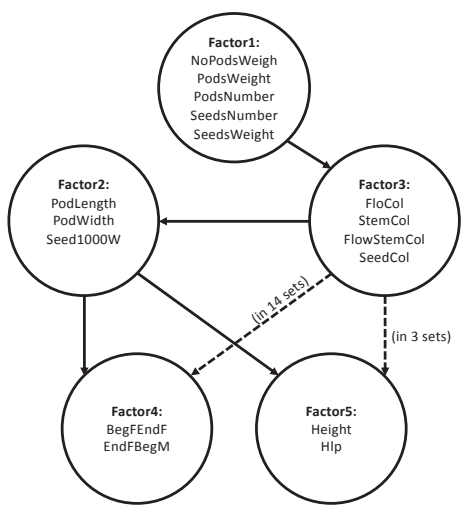

Fig 3. Latent factors joined to form structural part of connected SEM model. Dashed arrows represent relationships, which were not present is all training sets for directed acyclic graph obtained; Solid lanes represent relationships, which were found in each of 20 training sets

Then, we tested historical hypotheses about chickpea dispersals and found that in 9 out of 10 populations, dispersals we likely occurred along with trade routes. We took allele frequency estimated obtained from 'trade route' hypothesis and get distributions off phenotypic traits in ancestral populations (centers of clusters), which were in line with origin of light chickpea subtype (kabuli).

\section{ACKNOWLEDGMENT}

The project is supported by the RBFR grant 18-29-13033.

\section{REFERENCES}

[1] Igolkina AA, Meshcheryakov G. semopy: A Python Package for Structural Equation Modeling. Struct Equ Model A Multidiscip doi:10.1080/10705511.2019.1704289.

[2] Igolkina A, Meshcheryakov G, Gretsova M, Nuzhdin S, Samsonova M. Multi-trait multi-locus SEM model discriminates SNPs of different effects. 2020.

[3] Pawlowsky-Glahn V, Buccianti A. Compositional Data Analysis. Chichester, UK: John Wiley \& Sons, Ltd; 2011. doi:10.1002/9781119976462. 